\title{
EVALUATION OF 48-WEEK DAILY TERIPARATIDE TREATMENT WITH DXA-BASED HIP STRUCTURE ANALYSIS IN ELDERLY FEMALE PATIENTS WITH RHEUMATOID ARTHRITIS
}

Sho Mokuda, ${ }^{1,2} *$ Minoru Fujita ${ }^{2}$, Naoya Sawada ${ }^{1}$, Yosuke Murata ${ }^{1}$, Kenichiro Matoba ${ }^{1}$, Akihiro Yamada ${ }^{1}$, Makoto Onishi ${ }^{1}$, Yasuaki Okuda ${ }^{1}$, Kazuo Jouyama and Kiyoshi Takasugi ${ }^{1}$

${ }^{1}$ Department of Internal Medicine, Center for Rheumatic Diseases, Dohgo Spa Hospital, Matsuyama, Ehime, Japan

${ }^{2}$ Department of Radiology, Center for Rheumatic Diseases, Dohgo Spa Hospital,

Matsuyama, Ehime, Japan

\section{*Address for corresponding} author: Sho Mokuda, MD Department of Clinical immunology and Rheumatology Hiroshima University Hospital,

1-2-3 Kasumi, Minami-ku, Hiroshima, 734-8551, Japan Telephone: $+81-82-257-5178$ Fax: +81-82-257-5179 E-mail: sho-mokuda@hiroshima-u.ac.jp

\begin{abstract}
Objectives: The purposes of the present study were to clarify the effects on the structural strength of the femoral neck bones in elderly RA patients being treated with teriparatide.

Methods: Fifty female RA patients were enrolled. We investigated the bone mineral density (BMD) of the lumbar vertebrae and femoral neck, and performed a hip structure analysis (HSA) using dual-energy X-ray absorptiometry (DXA) during a 48-week treatment with teriparatide (20 $\mu \mathrm{g} /$ day).
\end{abstract}

Results: The mean age was 74.5 years, and the mean duration of RA was 23.0 years. The percent change in the lumbar BMD was $+5.8 \%$ (mean, 24 weeks, $P<0.01$ ) and $+8.0 \%$ (mean, 48 weeks, $P<0.01$ ). On the other hand, there were no statistically significant differences in the femoral neck BMD changes at either 24 or 48 weeks $(P=0.729$ and $P=0.567$, respectively). The percentage change from baseline in the buckling ratio of the femoral neck was $16.8 \%$ (improve) at 48 weeks $(P=0.004)$, because of cortical thickening. There were no significant differences in the section modulus (SM) or cross-sectional area (CSA).

Conclusions: The femoral neck bone strength increased with teriparatide treatment in elderly female RA patients.

Key Words: DXA-based hip structure analysis, glucocorticoid, osteoporosis, rheumatoid arthritis, teriparatide. 


\section{INTRODUCTION}

Patients with rheumatoid arthritis (RA) often suffer from osteoporosis (OP). $\mathrm{OP}$ is one of the extra-articular complications of RA, and results in an increased risk of fractures (Roux C 2011). RA is a chronic, systemic, inflammatory disease with polyarthritis, and multiple factors (for example, immobility, cytokines, inflammation around joints, and treatment with corticosteroids, etc.) contribute to the generalized osteoporosis in RA patients (Roux C 2011, Vis $M$ et al. 2013). Longitudinal studies in RA patients have shown reduced bone mineral density (BMD) and several-fold increases in the relative risk for spine and hip fractures

(Ørstavik RE et al. 2004, Haugeberg G et al. 2000, van Staa TP et al. 2006, Amin S, et al. 2013). However, there have so far been few controlled trials of antiosteoporotic drugs, including teriparatide, in RA patients.

Teriparatide (the recombinant $\mathrm{N}$ terminal fragment of human parathyroid hormone, rhPTH(1-34)) is a PTH analogue with bone-anabolic actions. In postmenopausal females with osteoporosis, once-daily teriparatide increased BMD and reduced the risk of vertebral and nonvertebral fractures in a placebo control study (Neer RM, et al. 2001). In another placebo control study in male patients, teriparatide improved BMD and reduced the risk of vertebral fractures (Kaufman $\mathrm{JM}$, et al. 2005). Moreover, in a randomized, controlled trial, patients with glucocorticoid-induced osteoporosis (GIO) treated with teriparatide had greater increases in their lumbar spine BMD and fewer new vertebral fractures than subjects treated with alendronate (Saag KG, et al. 2007. Saag KG, et al. 2009). A prospective observational study revealed that teriparatide improved BMD in RA patients (Ebina K, et al. 2014).

The purposes of the present study were to clarify its effects on BMD and the femoral neck bone strength treated for 48 weeks in RA patients with osteoporosis. In this survey, we performed a dual energy Xray absorptiometry (DXA) based hip structure analysis (HSA) to evaluate the effects of the treatment on the bone strength in RA patients. The DXA-based HSA method was noninvasive, and was able to measure the strength of bone governed by structural dimensions and tissue mineral properties (Beck TJ, et al. 1990). This method was performed to evaluate the effects of anti-osteoporotic drugs, for example, alendronate, risedronate, minodronate, raloxifene and teriparatide (Bonnick SL, et al. 2009, Takada J, et al. 2010, Ito M, et al. 2010, Takada J, et al. 2011, Urusi-Rasi K, et al. 2005). DXAbased HSA had already been used to demonstrate that teriparatide treatment increased the cortical thickness and stability at the femoral neck region in primary osteoporotic females (Urusi-Rasi K, et al. 2005).

\section{MATERIALS AND METHODS}

\subsection{Study population and protocol}

This study was a single center observational study and was performed from October 2010 through May 2013. This study was approved by the clinical ethics committees of Dohgo Spa Hospital. We obtained informed consent from all 40 RA patients before the treatment with 48-weeks daily teriparatide, and collected their clinical data. All patients with rheumatoid arthritis fulfilled the classification criteria of the ACR 1987 (Arnett FC, et al. 1988). The exclusion criteria included the patients who were male, those receiving corticosteroid 
pulse therapy and non-osteoporotic patients.

When patients with osteoporosis started treatment with percutaneous daily teriparatide $(20 \mu \mathrm{g} /$ day $)$, they discontinued their previous treatment for osteoporosis (for example, bone resorption inhibitors (BRI) (e.g. bisphosphonates, selective estrogen receptor modulators), the active form of vitamin D3, etc.) and started to take a calcium supplement (one gram, daily) as a concomitant treatment. The background data of the patients were collected from each medical record, and included the patient's age, body mass index (BMI), retrospective disease history and duration, retrospective history of using antiosteoporotic drugs and history of antirheumatic drugs. The following data were also collected: 1$)$ the serum creatinine $(\mathrm{Cr})$, serum albumin level, serum calcium level and serum phosphate level, serum intactparathyroid hormone (intact-PTH) levels and digitized thoracolumbar X-rays at baseline ( 0 weeks, before treatment $), 2$ ) the lumbar and femoral neck BMD and DXAbased HSA at 0 weeks, 24 weeks and 48 weeks. The eGFR (estimated glomerular filtration rate) was calculated using the following formula devised by the Japanese Society of Nephrology: eGFR $(\mathrm{mL} / \mathrm{min} / 1.73 \mathrm{~m} 2)=194 \times$ Cr-1.094 x Age$0.287 \times 0.739$. The corrected calcium value was calculated using the following formula: Corrected calcium $(\mathrm{mg} / \mathrm{dL})=$ serum calcium $(\mathrm{mg} / \mathrm{dL})+(4-$ serum albumin $(\mathrm{g} / \mathrm{dL}))$.

2.2 Bone mineral density (BMD) and DXAbased hip structure analysis (HSA)

DXA scans performed with the Lunar DPX-BRAVO bone densitometer (GE Healthcare Japan, Co., Ltd., Tokyo, Japan). Hip scan image data were analyzed using the "Advanced Hip Assessment Copyright @ 2015, Knowledge Enterprises Incorporated. All rights reserved.
(AHA)" software program (GE Healthcare Japan, Co., Ltd., Tokyo, Japan) and were conducted by the same technician (M.F.) at Dohgo Spa Hospital. The AHA program automatically calculates a number of bone geometry variables from the scan image and bone distribution variables derived from information contained within the DXA Xray absorption curves. In this program, we could not measure the intertrochanteric structures, femoral shaft structures, outer and inner diameters for narrow-neck. The HSA geometry algorithm is based on a principle first articulated by Martin and Burr (Martin R and Burr D 1984). In this study, we analyzed with buckling ratio (BR), cortical thickness, section modulus (SM) and cross-section area (CSA) of femoral neck of the femoral narrow-neck. The BR is a crude index of the susceptibility to local buckling under bending loads. The SM and CSA are indexes of resistance to axial compressive and bending loads.

\subsection{Radiographic measurements of vertebral fractures}

To evaluate an existing fracture (prevalent fracture), we used a semiquantitative (SQ) thoracolumbar Xrays-based method (Genant $\mathrm{HK}$, et al. 1993). If a vertebral bone (Th4-L4) has an osteoporotic deformation with an SQ grade $\geq 1$, we concluded that the bone was fractured.

\subsection{Diagnosis of osteoporosis}

Osteoporosis was defined by the following: a T-score $\leq-2.5$ or an existing osteoporotic fracture, referencing the World Health Organization (WHO) definition of osteoporosis based on BMD (Kanis JA, et al. 1994). 


\section{$2.5 R A$ disease activity}

The RA disease activity was calculated based on the DAS28-ESR according to the formula on the DAS (Disease Activity Score) web site (http://www.das-score.nl/index.html).

\subsection{Statistical analysis}

The normality of the data was assessed using the chi-square test. The Wilcoxon signed ranks test was applied for continuous variables, and the Chi-square test was applied for categorical variables. The Spearman rank correlation test was used to assess relationships between the buckling ratio (femoral neck) change and other factors. The data processing and analyses were conducted using the Microsoft Excel software program.

\section{RESULTS}

\subsection{Baseline characteristics and tolerability of daily teriparatide treatment in $R A$} patients

Forty RA patients with osteoporosis were included in this study. Table 1 lists the baseline characteristics and biochemical parameters for these patients (Table 1). The mean age ( \pm standard deviation $(\mathrm{SD})$ ) was $74.5 \pm 7.6$ years, and the mean duration was $23.0 \pm 12.8$ years. The serum calcium level, phosphorus level and intact-PTH level were within the normal ranges in most cases.

\subsection{Changes in the lumbar and femoral neck $B M D$}

The percentage changes from baseline in the lumbar and femoral neck BMD are noted in Table 2. After the 24 and 48 weeks of treatment with teriparatide, the lumbar BMD showed significant increases (24 weeks (mean), +5.8\%; 48 weeks Copyright @ 2015, Knowledge Enterprises Incorporated. All rights reserved. (mean), $+8.0 \%$ ) (24 weeks: $P=0.0001 ; 48$ weeks: $P=0.0001)$. On the other, there were no statistically significant differences in the femoral neck BMD changes at 24 and 48 week ( 24 weeks (mean), $+2.3 \%$; 48 weeks (mean), -0.1\%) (24 weeks, $P=0.729 ; 48$ weeks, $P=0.567)$.

\subsection{Changes in indexes from DXA-based HSA}

We also performed a DXA-based HSA to estimate the femoral neck bone strength in the RA patients (Table 2). As a result, we found that the percentage changes from baseline in the buckling ratio (BR) and cortical thickness were $-16.8 \%$ (mean) and $+34.3 \%$ (mean) at 48 weeks $(P=0.004$, $P=0.039)$. There was no significant different in the section modulus (SM) or cross-section area (CSA) at 48 weeks $(P=0.899, \quad P=0.797)$. Therefore, the teriparatide treatment did not change the femoral neck BMD in RA patients, but did improve the femoral neck bone strength.

Moreover, the Spearman rank correlation test was used to assess the relationships between the buckling ratio (femoral neck) change at 48 weeks and other factors, including the age, BMI, RA disease duration, DAS28-ESR, prednisolone dose at baseline, prior use of BRI and prevalent vertebral fractures (Table 3). Consequently, the daily prednisolone dose at baseline positively correlated with the buckling ratio ( $r s=0.402, P=0.049$ ). 
Table1. Baseline characteristics of RA patients treated with 48-weeks teriparatide

RA treated with 48-weeks TPTD

Characteristic

Age, mean (SD), years

BMI, mean (SD), $\mathrm{kg} / \mathrm{m} 2$

RA disease duration, mean (SD), years

Prednisolone dose, median (IQR), mg/day

Prior use of bone resorption inhibitors

DAS28-ESR, mean (SD)

Estimated GFR, mean (SD), $\mathrm{mL} / \mathrm{min} / 1.73 \mathrm{~m} 2$

Corrected calcium level, mean (SD), mg/dL

Serum phosphorus level, mean (SD), mg/dL

BMD (lumbar spine (L2-4)), median (IQR), g/cm2

BMD (femoral neck), mean (SD), g/cm2

Cortical thickness (femoral neck), mean (SD), mm

Buckling ratio (femoral neck), median (IQR)

Section modulus (SM) (femoral neck), mean (SD), mm3

Cross-sectional area (CSA) (femoral neck), mean (SD), mm2
$74.5(7.6)$

$20.6(3.7)$

$23.0(12.8)$

$5.0(4.0-5.0)$

$27 / 40(67.5 \%)$

$2.43(0.74)$

$80.4(29.8)$

$9.2(0.46)$

$3.5(0.46)$

$0.834(0.717-0.966)$

$0.54(0.15)$

$2.1(0.91)$

$8.55(6.48-11.50)$

$287(81.0)$

78.3 (16.6)

SD, standard deviation; IQR, ESR, Erythrocyte sedimentation rate; GFR, interquartile range; RA, rheumatoid Glomerular filtration rate. arthritis; BMD, Bone Mineral Density;

Table 2. The percent changes from baseline for the BMD and DXA-based HSA

\begin{tabular}{ccc}
\hline (Mean \pm SE) & 24 weeks $(\%)$ & 48 weeks $(\%)$ \\
\hline Lumbar BMD (L2-4) & $5.8 \pm 1.5 * *$ & $8.0 \pm 2.0 * *$ \\
Femoral neck BMD & $2.3 \pm 3.1$ & $-0.1 \pm 1.9$ \\
Cortical thickness & $13.4 \pm 9.7$ & $34.3 \pm 16.5 *$
\end{tabular}




$\begin{array}{ccc}\text { Buckling ratio (BR) } & -1.4 \pm 8.0 & -16.8 \pm 5.9 * *, \text { (a) } \\ \text { Section modulus (SM) } & 0.13 \pm 2.2 & 1.3 \pm 3.3 \\ \text { Cross-sectional area (CSA) } & -1.8 \pm 1.7 & -1.6 \pm 2.3\end{array}$

BMD, Bone Mineral Density; SE, standard error.

(a) The buckling ratio was improved at 48 weeks.

Wilcoxon's signed rank test, ${ }^{* *} P<0.01, * P<0.05$.

Table 3. The results of the univariate analysis of vertebral fracture progression and a worsening buckling ratio

Percent change of buckling ratio

Risk factor

rs $P$-value

\begin{tabular}{llc}
\hline Age & 0.038 & 0.850 \\
Body mass index & 0.018 & 0.927 \\
RA disease duration & -0.053 & 0.775 \\
DAS28-ESR & 0.025 & 0.899 \\
Prednisolone dose at 0-week & 0.402 & $0.049^{*}$ \\
Prior usage of bone resorption & -0.082 & 0.067 \\
inhibitors & & \\
Number of prevalent vertebral & & 0.849 \\
fracture (Th4-L4) & -0.027 & \\
\hline
\end{tabular}

RA, rheumatoid arthritis; BMD, Bone Mineral Density; ESR, Erythrocyte sedimentation rate; rs, Spearman's rank correlation coefficient.

* $P<0.05$ based on the Spearman rank correlation test

\section{DISCUSSION}

In the present study, we measured the response of teriparatide with BMD and DXA-based HSA. After 48 weeks of teriparatide treatment, the lumbar BMD levels increased and the femoral neck BMD levels was not elevated in patients with RA. In the EUROFORS study, the femoral neck BMD did not increase after 12 months of teriparatide treatment in patients who had been pretreated with BRI (Boonen S, et al. 2008), so our results might not be contradictory to this study, because $63.5 \%$ 
of the cases had prior BRI usage. However, one of the problems with the use of the BMD to monitor osteoporosis treatment is that it does not completely capture the mechanical factors that lead to fragility ( $\mathrm{Li}$ Z, et al. 2001, Cummings SR, et al. 2002, Sarkar S, et al. 2002). One of the main characteristics of teriparatide is that it has an anabolic effect, which changes the amount and distribution of bone material within a bone, thereby altering the geometrical structure. Therefore, we also used the DXA-based HSA to assess the femoral neck bone strength. As a result, the HSA showed that treatment with teriparatide improved the buckling ratio (BR) at 48 weeks. The elevation of the BR level caused by cortical thickening. UusiRasi et al. reported that the improvement of BR was observed in patients with primary osteoporosis after one year of treatment with teriparatide (Uusi-Rasi K, et al. 2005), which was in agreement with our present findings. While, there were no significant differences in the section modulus (SM) or cross-section area (CSA) during the 48week treatment period. In AHA software program, we were not able to analyze the other parameters, including outer and inner femoral diameters. Therefore, we could not discuss the discrepancy.

The univariate analyses showed that the daily prednisolone dose was associated with the buckling ratio, with higher doses being associated with less improvement. Therefore, the glucocorticoid dose should be kept as low as possible to maximize the impact of the teriparatide treatment.

Limitations. In this study, we cannot acquire the appropriate control group. Therefore, further studies, such as randomized trials, are needed to confirm the effect of teriparatide treatment in RA patients. And, we researched the effect of teriparatide in 48 weeks. Recently, in our country, teriparatide treatment for osteoporosis is able to continue in 96 weeks.

Conclusions. Treatment of teriparatide in elderly female RA patients with osteoporosis has the potential to increase the femoral neck bone strength, even if the femoral neck BMD is not elevated. 


\section{REFERENCES}

Amin S, Gabriel SE, Achenbach SJ, Atkinson EJ, Melton LJ 3rd: Are young women and men with rheumatoid arthritis at risk for fragility fractures? A populationbased study. J Rheumatol. 2013; 40: 166976.

Arnett FC, Edworthy SM, Bloch DA, McShane DJ, Fries JF, Cooper NS, et al.: The American Rheumatism Association 1987 revised criteria for the classification of rheumatoid arthritis. Arthritis Rheum. 1988; 31: 315-24.

Beck TJ, Ruff CB, Warden KE, Scott WW Jr, Rao GU: Predicting femoral neck strength from bone mineral data. A structural approach. Invest Radiol. 1990; 25: 6-18.

Bonnick SL, Beck TJ, Cosman F, Hochberg MC, Wang H, de Papp AE: DXA-based hip structural analysis of once-weekly bisphosphonate-treated postmenopausal women with low bone mass. Osteoporos Int. 2009; 20: 911-21.

Boonen S, Marin F, Obermayer-Pietsch B, Simões ME, Barker C, Glass EV, et al.:

Effects of previous antiresorptive therapy on the bone mineral density response to two years of teriparatide treatment in postmenopausal women with osteoporosis. J Clin Endocrinol Metab. 2008; 93: 852-60.

Cummings SR, Karpf DB, Harris F, Genant HK, Ensrud K, LaCroix AZ, et al.: Improvement in spine bone density and reduction in risk of vertebral fractures during treatment with antiresorptive drugs. Am J Med. 2002; 112: 281-9.

Ebina K, Hashimoto J, Shi K, Kashii M,
Hirao M, Yoshikawa H. Comparison of the effect of 18-month daily teriparatide administration on patients with rheumatoid arthritis and postmenopausal osteoporosis patients. Osteoporos Int. 2014; 25: 275565.

Genant HK, Wu CY, van Kuijk C, Nevitt MC: Vertebral fracture assessment using a semiquantitative technique. J Bone Miner Res. 1993; 8: 1137-48.

Haugeberg G, Uhlig T, Falch JA, Halse JI, Kvien TK: Bone mineral density and frequency of osteoporosis in female patients with rheumatoid arthritis: results from 394 patients in the Oslo County Rheumatoid Arthritis register. Arthritis Rheum. 2000; 43: 522-30.

Ito $\mathrm{M}$, Sone $\mathrm{T}$, Fukunaga M: Effect of minodronic acid hydrate on hip geometry in Japanese women with postmenopausal osteoporosis. J Bone Miner Metab. 2010; 28: 334-41.

Kanis JA, Melton LJ III, Christiansen C, Johnston CC, Khaltaev N: The diagnosis of osteoporosis. J Bone Miner Res. 1994; 9: 1137-41.

Kaufman JM, Orwoll E, Goemaere S, San Martin J, Hossain A, Dalsky GP, et al.: Teriparatide effects on vertebral fractures and bone mineral density in men with osteoporosis: treatment and discontinuation of therapy. Osteoporos Int. 2005; 16: 5106.

Li Z, Meredith MP, Hoseyni MS: A method to assess the proportion of treatment effect explained by a surrogate endpoint. Stat Med. 2001; 20: 3175-88.

Martin R, Burr D. Non-invasive measurement of long bone cross-sectional 
moment of inertia by photon absorptiometry. J Biomech 1984; 17: 195201.

Neer RM, Arnaud CD, Zanchetta JR, Prince R, Gaich GA, Reginster JY, et al.: Effect of parathyroid hormone (1-34) on fractures and bone mineral density in postmenopausal women with osteoporosis. N Engl J Med. 2001; 344: 1434-41.

Ørstavik RE, Haugeberg G, Mowinckel P, Høiseth A, Uhlig T, Falch JA, et al.:

Vertebral deformities in rheumatoid arthritis: a comparison with populationbased controls. Arch Intern Med. 2004; 164: 420-5.

Roux C. Osteoporosis in inflammatory joint diseases. Osteoporos Int. 2011; 22: 421-33.

Saag KG, Shane E, Boonen S, Marín F,

Donley DW, Taylor KA, et al.: Teriparatide or alendronate in glucocorticoid-induced osteoporosis. N Engl J Med. 2007; 357: 2028-39.

Saag KG, Zanchetta JR, Devogelaer JP, Adler RA, Eastell R, See K, et al.: Effects of teriparatide versus alendronate for treating glucocorticoid-induced osteoporosis: thirty-six-month results of a randomized, double-blind, controlled trial. Arthritis Rheum. 2009; 60: 3346-55.

Sarkar S, Mitlak BH, Wong M, Stock JL, Black DM, Harper KD: Relationships between bone mineral density and incident vertebral fracture risk with raloxifene therapy. J Bone Miner Res. 2002; 17: 1-10.

Takada J, Miki T, Imanishi Y, Nakatsuka $\mathrm{K}$, Wada $\mathrm{H}$, Naka $\mathrm{H}$, et al.: Effects of raloxifene treatment on the structural geometry of the proximal femur in
Japanese women with osteoporosis. J Bone Miner Metab. 2010; 28: 561-7.

Takada J, Katahira G, Iba K, Yoshizaki T, Yamashita T: Hip structure analysis of bisphosphonate-treated Japanese postmenopausal women with osteoporosis. J Bone Miner Metab. 2011; 29: 458-65.

Uusi-Rasi K, Semanick LM, Zanchetta JR, Bogado CE, Eriksen EF, Sato M, et al.: Effects of teriparatide [rhPTH (1-34)] treatment on structural geometry of the proximal femur in elderly osteoporotic women. Bone. 2005; 36: 948-58.

van Staa TP, Geusens P, Bijlsma JW, Leufkens HG, Cooper C: Clinical assessment of the long-term risk of fracture in patients with rheumatoid arthritis. Arthritis Rheum. 2006, 54: 3104-12.

Vis M, Güler-Yüksel M, Lems WF. Can bone loss in rheumatoid arthritis be prevented? Osteoporos Int. 2013; 24: 254153. 